\title{
INVESTIGATION OF MATTER ENHANCED NEUTRINO OSCILLATIONS RELEVANT TO THE SOLAR NEUTRINO PROBLEM
}

\author{
J.M. LOSECCO ${ }^{a}$, R.M. BIONTA ${ }^{b}$, G. BLEWITT ${ }^{c}$, C.B. BRATTON ${ }^{d}$, D. CASPER $^{b}$, R. CLAUS $^{b}$, \\ B. CORTEZ ${ }^{c}$, S. ERREDE ${ }^{b}$, G. FOSTER $^{b}$, W. GAJEWSKI ${ }^{\mathrm{e}}$, K.S. GANEZER $^{\mathrm{e}}$, M. GOLDHABER $^{\mathrm{f}}$, \\ T.J. HAINES ${ }^{\mathrm{e}}$, T.W. JONES ${ }^{\mathrm{g}}$, D. KIELCZEWSKA ${ }^{\text {h }}$, W.R. KROPP e , J.G. LEARNED ${ }^{\mathrm{i}}$, E. LEHMANN ${ }^{\mathrm{c}}$, \\ H.S. PARK ${ }^{b}$, F. REINES ${ }^{\text {e }}$, J. SCHULTZ ${ }^{\mathrm{e}}$, S. SEIDEL ${ }^{\mathrm{b}}$, E. SHUMARD ${ }^{\mathrm{b}}$, D. SINCLAIR ${ }^{\mathrm{b}}$, H.W. SOBEL $^{\mathrm{e}}$, \\ J.L. STONE ${ }^{b}$, L. SULAK ${ }^{b}$, R. SVOBODA ${ }^{i}$, J.C. VAN DER VELDE ${ }^{b}$ and C. WUEST ${ }^{\mathrm{e}}$ \\ a University of Notre Dame, Notre Dame, IN 46556, USA \\ ${ }^{b}$ University of Michigan, Ann Arbor, MI 48109, USA \\ ${ }^{c}$ California Institute of Technology, Pasadena, CA 91125, USA \\ ${ }^{d}$ Cleveland State University, Cleveland, OH 44115, USA \\ $e$ University of California, Irvine, CA 92717, USA \\ $f$ Brookhaven National Laboratory, Upton, NY 11973, USA \\ $g$ University College, London WC1E $8 B T, U K$ \\ $h^{h}$ Warsaw University, PL-00-681 Warsaw, Poland \\ ${ }^{i}$ University of Hawaii, Honolulu, HI 96822, USA
}

Received 20 October 1986

\begin{abstract}
We study the effect of matter enhanced neutrino oscillations on atmospheric neutrinos. A recently proposed solution to the solar neutrino problem with $\Delta m^{2}=1.1 \times 10^{-4} \mathrm{eV}^{2}$ suggests enhanced effects in the range $200 \mathrm{MeV}-500 \mathrm{MeV}$. We find no evidence of this effect for $\nu_{\mu} \leftrightarrow \nu_{\mathrm{e}}$ mixing. Limits are set on the magnitude of the mixing angle. Our limit is $\sin \theta_{\mathrm{V}}<0.14$ at $90 \%$ confidence level. The limit is dominated by statistical errors and may be improved.
\end{abstract}

It has recently been proposed [1] that the solar neutrino problem may be explained through matter enhanced neutrino oscillations. Under this hypothesis the electron neutrinos generated from ${ }^{8} \mathrm{~B}$ decay in the center of the sun get converted to non-interacting neutrinos, $\nu_{\mu}$ or $\nu_{\tau}$, as they traverse the sun. This solution requires a mass difference of $1.1 \times 10^{-4} \mathrm{eV}^{2}$ between the neutrino that is dominantly $\nu_{\mathrm{e}}$ and the other that is dominantly $\nu_{\mu}$ or $\nu_{\tau}$. Such a $\Delta m^{2}$ can only be studied in terrestrial experiments using atmospheric neutrinos [2].

The mean density of the earth is $5.5 \mathrm{gm} / \mathrm{cm}^{3}$. On the basis of the solar neutrino result one would expect large oscillation effects for neutrinos of energy $275 \mathrm{MeV}$ traversing the earth. Due to variations in the earth's density and the width of the "resonance" large effects will occur for a range of energies (fig. 1).

Even for the "resonant" energy, where the amplitude for conversion eventually equals $100 \%$ the oscillation length is inversely proportional to the mixing angle [2]:

$l_{\mathrm{M}}=2 \pi E_{\nu} / \Delta m^{2} \sin 2 \theta_{\mathrm{v}}$,

with $E_{\nu}$ in $\mathrm{MeV}, \Delta m^{2}$ in $\mathrm{eV}^{2}$ and $l_{\mathrm{M}}$ in $\mathrm{m}$.

The greatest sensitivity to small $\theta_{\mathrm{v}}$ is obtained for the largest path length. In the present experiment that is achieved for near vertical, upward going neutrino interactions. Even then there are very small values of $\sin 2 \theta_{\mathrm{v}}$ that will have such a large oscillation length that effects will not be measurable. The solar solution is satisfied for any $\theta_{\mathrm{v}}>0.0065$.

The atmospheric neutrino flux is a classical mixture of neutrino and antineutrino and of muon and electron type neutrinos. Measurements indicate that muon neutrinos outnumber electron neutrinos by about a factor of 
3.1. The neutrino flux is about $25 \%$ higher than the antineutrino flux. This and cross section differences give a factor of 3 excess for neutrino interactions over antineutrino interactions.

A solution to the solar neutrino problem requires the electron neutrino to mix with another neutrino. In general it is assumed to be the muon neutrino. A number of possibilities exist. If the electron neutrino mixes with the muon neutrino then both recordable $\nu$ fluxes will be affected. Due to the differences in muon and electron detection, enhanced muon to electron neutrino oscillations may be studied with relative ease. On the other hand if the electron mixes with the tau neutrino the only signature available at these energies will be a drop in electron neutrino interactions. Since they represent a small fraction of all events sensitivity will be limited.

Here we will study the effects of electron to muon neutrino oscillations. Since our source is a mixture of both kinds of neutrinos we must take into account conversion in both directions. A very sensitive experiment is possible taking into account the two different detection thresholds for the two different neutrinos at the energies of interest. Conversion of a $\nu_{\mu}$ to a $\nu_{\mathrm{e}}$ would give a dramatic increase in event rate. There is also some ability to distinguish $\nu_{\mu}$ from $\nu_{\mathrm{e}}$ by the decay of a muon in the final state.

Since the earth is nonuniform we must carry out a numerical integration to determine the effects of the matter. We work in the electron-muon basis ${ }^{\ddagger 1}$. For a particular choice of energy $\left(E_{\nu}\right)$, direction, mixing angle $\left(\sin \theta_{\mathrm{V}}\right)$ and mass parameter $\left(\Delta m^{2}\right)$ we integrate to find the wave function through the earth.

$\left(\begin{array}{l}\dot{\nu}_{\mathrm{e}} \\ \dot{\nu}_{\mu}\end{array}\right)=-\mathrm{i}\left(\begin{array}{ll}\left(\Delta m^{2} / 2 \hbar c E_{\nu}\right) \sin ^{2} \theta_{\mathrm{v}}+\sqrt{2} G N_{\mathrm{A}} \frac{1}{2} \rho(x) & \left(\Delta m^{2} / 2 \hbar c E_{\nu}\right) \sin \theta_{\mathrm{v}} \cos \theta_{\mathrm{v}} \\ \left(\Delta m^{2} / 2 \hbar c E_{\nu}\right) \sin \theta_{\mathrm{v}} \cos \theta_{\mathrm{v}} & \left(\Delta m^{2} / 2 \hbar c E_{\nu}\right) \cos ^{2} \theta_{\mathrm{v}}\end{array}\right)\left(\begin{array}{l}\nu_{\mathrm{e}} \\ \nu_{\mu}\end{array}\right)$,

where $\rho(x)$ is the density of matter at point $x \cdot \frac{1}{2} \rho(x)$ is the electron density in normal matter.

We have used $\Delta m^{2}=1.1 \times 10^{-4} \mathrm{eV}^{2}$ as suggested by the solar neutrino solution [1]. Small mixing angles were investigated. Due to poor directional resolution and due to the low counting rate the direction was integrated over large regions of solid angle. Since the distribution of matter inside the earth is not well known we have studied three possible models $[3,4]$.

The three models differ only in the mass density distribution near the center of the core, varying from $13 \mathrm{gm} /$ $\mathrm{cm}^{3}$ to $21 \mathrm{gm} / \mathrm{cm}^{3}$. These differences have a negligible effect on our result. The differences subtend a very small solid angle as seen by the detector (a region of about $10^{\circ}$ from the upward vertical. Even neutrinos going directly through this region spend a very small portion of the total path length there; at most $2000 \mathrm{~km}$ out of $13000 \mathrm{~km}$. The only discernable effect is to make slight differences in the energy $(\approx 20 \mathrm{MeV})$ at which maximum oscillations occur. The detector's energy resolution is poor compared to these shifts and we have integrated over energy in our analysis anyway. Our data is integrated over $20 \%$ of all solid angle. This is large compared to the $1 \%$ ambiguous region. Analyses carried out with all three yield comparable results. We have made an effort to keep the density distribution $\rho(x)$ smooth and continuous in our integration.

We employ the 401 contained event sample [5] of the 3300 metric ton IMB imaging water Cerenkov detector. The device records the direction, energy and multiplicity of atmospheric neutrino interactions. A number of details limit the sensitivity. A threshold of $200 \mathrm{MeV}$ in visible energy is required to reconstruct the events. This corresponds to $200 \mathrm{MeV}$ for an electron neutrino but to $450 \mathrm{MeV}$ for muon neutrinos due to the energy lost to the muon mass. The detector can not observe tracks below the Čerenkov threshold $\left(1.52 \times m c^{2}\right)$ so at best a lower limit on the energy is recorded. This means that at low visible energies a significant fraction of the events come from neutrinos with higher energies. These higher energies can contribute to the background of an oscillation signal but are much less sensitive to the presence of an effect. To a large extent detector related effects including efficiencies can be taken into account by comparing data going upward which could manifest an oscillation effect with that coming downward which cannot.

If $\nu_{\mu} \leftrightarrow \nu_{\mathrm{e}}$ oscillations occur they would manifest themselves as a net increase in rate of electron neutrino interactions due to the much larger source of muon neutrinos. The lower energy threshold for electron neutrino inter-

${ }^{\ddagger 1}$ We would like to thank S.P. Rosen for help in formulating the equation. 
actions would give rise to a net increase in the overall event rate.

Only a small portion of the data is sensitive to matter enhanced oscillations with this $\Delta m^{2}$. Cuts have been chosen to maximize the number of events in the $E_{\nu}<400 \mathrm{MeV}$ region that have travelled on the longest path through the earth. We have selected events within $53^{\circ}$ of the vertical going either up or down. These represent two samples containing $20 \%$ of the solid angle each. The upward sample has a mean path length of $10.2 \times 10^{6} \mathrm{~m}$. To ensure good directions we have selected single-prong events. Since the effect only influences low energy events (fig. 1) we have required the visible energy to be less than $350 \mathrm{MeV}$. This gives us a sample of 18 events going upward and 25 going downward:

up/down $=0.72 \pm 0.22 \leqslant 1.00 \quad(90 \%$ confidence limit $)$.

We can remove some of the $\nu_{\mu}$ interactions, enriching the sample even more by looking for a muon decay signature within $7.5 \mu \mathrm{s}$ after the interaction has occurred. The efficiency for finding such a signal is about $50 \%$. This cut leaves us with 14 events going upward and 18 going downward:

up/down $=0.78 \pm 0.28 \leqslant 1.14 \quad(90 \%$ confidence limit $)$.

Monte Carlo studies indicate that the downward sample should have about $72 \%$ electron neutrino and antineutrino interactions. $57 \%$ of the $\nu_{\mathrm{e}}$ interactions had a parent neutrino with energy less than $400 \mathrm{MeV}$. Geomagnetic modulation [6] of the flux is significant at these energies. Such considerations would predict [6] a ratio of about 0.71 in good agreement with the data. We can achieve a bound on any excess rate and attribute all of it to $\nu_{\mathrm{e}}$ to get a conservative limit:

excess $\nu_{\mathrm{e}}=0.9 \pm 3.6$.

To get a bound on the rate of oscillations for $\nu_{\mu}$ into $\nu_{\mathrm{e}}$ we need an estimate for the flux of $\nu_{\mu}$ potentially capable of oscillating into $\nu_{\mathrm{e}}$. Since we are below the $\nu_{\mu}$ detection threshold we must calculate the $\nu_{\mu}$ flux from the downward observed $\nu_{\mathrm{e}}$ flux. Only $31 \%(72 \% \times 57 \% \times 75 \%)$ of the observed signal represents $\nu_{\mathrm{e}}$ below $400 \mathrm{MeV}$. If $\nu_{\mu}$ convert to $\nu_{\mathrm{e}}$ and some primary $\nu_{\mathrm{e}}$ convert to $\nu_{\mu}$ the sensitivity is reduced by the loss of the $\nu_{\mathrm{e}}$ events. This reduces the effective $\nu_{\mu}$ flux to 11.7 events. This gives the limit:

$R\left(\nu_{\mu} \leftrightarrow \nu_{\mathrm{e}}\right)=0.08 \pm 0.31 \leqslant 0.48 \quad(90 \%$ confidence level).

There are a number of systematic errors that might be present. The $\nu_{\mu} / \nu_{\mathrm{e}}$ ratio is uncertain. The effect of the muon decay requirement may not produce a sample with $72 \% \nu_{\mathrm{e}}$. Finally fluctuations may produce fewer than $57 \%$ low energy events in our sample. By comparing the results of a number of different experimental cuts on the data, including different solid angles, we estimate the sum of these errors is less than $20 \%$ in the rate and add it in quadrature with our statistical error above. This gives:

$R\left(\nu_{\mu} \leftrightarrow \nu_{\mathrm{e}}\right) \leqslant 0.55 \quad(90 \%$ confidence level $)$.

The region of parameter space excluded by this limit is determined by integrating the equations above in the energy range $200 \mathrm{MeV}<E_{\nu}<400 \mathrm{MeV}$. Fig. 1 shows the probability function for producing a $\nu_{\mathrm{e}}$ from a $\nu_{\mu}$ with the mixing angle $\sin \theta_{\mathrm{v}}=0.14$. The average value in the energy range under consideration is 0.55 . The figure represents the effects of averaging over all path lengths in the upward $20 \%$ of solid angle. Integrating in energy and in direction reduces sensitivity but is essential to obtain a statistically significant data sample. Fortunately the effects are quite large.

This result is significant even in the light of results already published [2]. The present analysis has included geomagnetic effects, effects of the uncertainty in the density distribution $\rho(x)$ and uncertainty in a number of systematic effects associated with the flux. These were neglected in the earlier analysis. Since this data sample is independent of that used in ref. [2] the results, in principle, could be combined.

These results may be improved in a number of ways. Better muon and electron separation would reduce the 


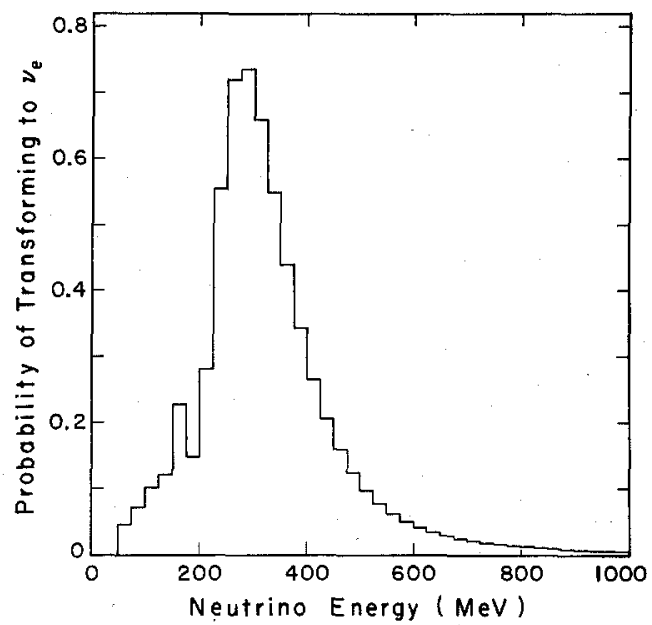

Fig. 1. The probability of producing an electron neutrino from a muon neutrino of a given energy after averaging over upward path lengths in $20 \%$ of the solid angle. The plot is for $\Delta m^{2}=$ $1.1 \times 10^{-4} \mathrm{eV}^{2}$ and for $\sin \theta_{\mathrm{V}}=0.14$.

contamination in the sample and enrich the signal. Total energy measurements would help remove the feed down from higher energies and give a pure low energy sample. We have assigned all of the possible excess to oscillations but in fact much of it could come from fluctuations in any of these additional sources. More data would reduce the statistical error that dominates our limit.

Our result can not rule out the solution of ref. [1]. We have constrained the magnitude of the mixing angle and this will be of use to model builders.

This work was supported in part by the US Department of Energy. One of us (JML) was supported in part by a DOE Outstanding Junior Investigator grant.

\section{References}

[1] H. Bethe, Phys. Rev. Lett. 56 (1986) 1305.

[2] J. LoSecco, Phys. Rev. Lett. 57 (1986) 652.

[3] C.W. Allen, Astrophysical quantities, third Ed. (Athlone Press, London, 1973) p. 118.

[4] B. Gutenberg, Physics of the earth's interior (Academic Press, New York, 1959) Ch. 7, and references therein.

[5] J. LoSecco et al., Phys. Rev. Lett. 54 (1985) 2299;

G. Blewitt et al., Phys. Rev. Lett. 55 (1985) 2114.

[6] T.K. Gaisser et al., Phys. Rev. Lett. 51 (1983) 223;

T.K. Gaisser and T. Stanev, private communication (October 1985). 